\title{
What If Dirac Pionini Existed in a Purely Chiral Superfield Formulation?
}

\author{
S. James Gates, Jr. 目 and Lubna Rana \\ Department of Physics \\ University of Maryland \\ College Park, MD 20742-4111 USA
}

\begin{abstract}
By an explicit construction, it is shown that the geometry of the $S U(3)$ pion multiplet with respect to the group manifold $S U_{L}(3) \otimes S U_{R}(3)$ may be deformed to admit a second pseudoscalar multiplet that is analogous to the $Z^{0}$ in unified theories of the electroweak interaction. This observation is found to play a key role in the construction of the $\mathrm{N}=1$ supersymmetric models with pions and Dirac-like spin-1/2 superpartners ('pionini').
\end{abstract}

\footnotetext{
${ }^{1}$ Supported in part by National Science Foundation Grant PHY-91-19746 and by NATO Grant CRG-93-0789

${ }^{2}$ gates@umdhep.umd.edu

${ }^{3}$ lubna@umdhep.umd.edu
} 


\section{Introduction}

In a recent series of papers [1, 2], an exploration of the possibility to construct $4 \mathrm{D}, \mathrm{N}=1$ superfield actions that generalize the phenomenological approach of Chiral Perturbation Theory [3] has been carried out. With the work of [2], the initial phase of the construction of super-Chiral Perturbation Theory (or "S-Chiral Perturbation Theory") was completed. Of course, there is no sign that the low-energy physics of pions is supersymmetric, but in a hypothetical world of unbroken supersymmetry, there is no impediment to the study of such a model.

In such a hypothetical world, accompanying the pion there would exist a set of spin-1/2 particles (hereafter to be called "pionini"). Carrying these consideration one step further, we are confronted with a choice of whether the pionini should be Dirac, Majorana or Weyl particles. This makes a big difference in the attempt to construct models of the QCD effective action in this hypothetical world. In the former case, it would be required that there are two $S U(3)$-octets of $4 \mathrm{D}, \mathrm{N}=1$ superfields to construct the supermultiplet containing the pionini. In the latter two cases, only one $S U(3)$-octet of $4 \mathrm{D}, \mathrm{N}=1$ superfields is required.

For our purposes, let us assume that the hypothetical pionini are Dirac particles. (One reason for this assumption is that most of the existing literature on $4 \mathrm{D}, \mathrm{N}=$ 1 supersymmetric QCD-like effective actions is based on the opposite assumption. Thus that case is well studied already.) On the other hand, it is only with our recent work [1] that models which describe Dirac pionini have entered the literature. However, the class of models that we have described so far utilizes both chiral (C), i.e. Wess-Zumino [6], and nonminimal (N) [7], i.e. complex linear, superfields to describe the two chiral components of a Dirac spinor. Since such a description uses both types of multiplets, we call these CNM (chiral-nonminimal multiplet) models. On the other hand, almost all of the literature on phenomenological applications of supersymmetry [8] uses pairs of chiral superfields to describe Dirac spinors. We are thus motivated to ask, "Whether a 4D, N = 1 supersymmetric QCD-like effective action containing Dirac pionini is possible to construct utilizing only chiral superfields?" The answer to this question turns out to be affirmative as we will see in this work.

As a by-product of this question posed within the context of supersymmetrical theories, we will also learn how it is possible to formulate the $S U_{L}(3) \otimes S U_{R}(3)$ geometry of the pion in a manner that has not (at least to our knowledge) appeared in the physics literature previously.

\footnotetext{
${ }^{4}$ For a nice review of a large part of the literature on this topic, see ref. 此. A discussion to consider more recent developments can be found in the work of ref. [5].
} 


\section{Ur-formulations of $S U_{L}(3) \otimes S U_{R}(3)$ Geometry of the Pion}

For as long as it has been known that the group manifold $S U_{L}(3) \otimes S U_{R}(3)$ plays an important role in understanding the dynamics of pions, it has been assumed the the interpretation of this particle in terms of the geometry of this manifold is unique. The pion octet usually appears as an element of the $S U(3)$ algebra in the form,

$$
\frac{1}{f_{\pi}} \Pi \equiv \frac{1}{f_{\pi}} \Pi^{i} t_{i}=\frac{1}{f_{\pi}}\left(\begin{array}{ccc}
\frac{\pi^{0}}{\sqrt{2}}+\frac{\eta}{\sqrt{6}} & \pi^{+} & K^{+} \\
\pi^{-} & -\frac{\pi^{0}}{\sqrt{2}}+\frac{\eta}{\sqrt{6}} & K^{0} \\
K^{-} & \bar{K}^{0} & -\eta \sqrt{\frac{2}{3}}
\end{array}\right)
$$

where $t_{1}, \ldots, t_{8}$ are essentially the Gell-Mann $S U(3)$ matrices and $f_{\pi}$ is the pion decay constant. The $S U(3)$ group elements are obtained via exponentiation $U \equiv$ $\exp \left[i \frac{1}{f_{\pi}} \Pi^{i} t_{i}\right]$. The rigid $S U_{L}(3) \otimes S U_{R}(3)$ transformations that are symmetries of the effective action are defined by

$$
(U)^{\prime}=\exp \left[-i \widetilde{\alpha}^{i} t_{i}\right] U \exp \left[i \alpha^{i} t_{i}\right]
$$

with independent real left and right transformation parameters $\widetilde{\alpha}^{i}$ and $\alpha^{i}$. For infinitesimal parameters this can be written as a variation of the $S U(3)$ octet containing the pion fields,

$$
\delta \Pi^{i}=-i f_{\pi}\left[\widetilde{\alpha}^{j}\left(L^{-1}\right)_{j}{ }^{i}-\alpha^{j}\left(R^{-1}\right)_{j}{ }^{i}\right] \equiv \alpha^{(A)} \xi_{(A)}^{i}
$$

We note that inverse Maurer-Cartan forms $\left(L^{-1}\right)_{j}{ }^{i}$ and $\left(R^{-1}\right)_{j}{ }^{i}$ appear. In finite form, this corresponds to the coordinate transformation,

$$
\left(\Pi^{i}\right)^{\prime}=K^{i}(\Pi)=\exp \left[\alpha^{(A)} \xi_{(A)}^{j} \partial_{j}\right] \Pi^{i} \quad, \quad \partial_{j} \equiv \partial / \partial \Pi^{j}
$$

Finally the $S U_{L}(3) \otimes S U_{R}(3)$ invariant action takes the form,

$$
\mathcal{S}_{\sigma}(\Pi)=-\frac{f_{\pi}^{2}}{2 C_{2}} \int d^{4} x \operatorname{Tr}\left[\left(\partial^{\underline{a}} U^{-1}\right)\left(\partial_{\underline{a}} U\right)\right] .
$$

Our notational conventions have been explained in the work of ref. [2].

Historically, the pion was introduced to be the fundamental mediator of the strong nuclear force much as the photon is the fundamental mediator of the electromagnetic force. Let us now carry this analogy a step (maybe too much) farther. With the construction of the Glashow-Weinberg-Salam model [9], it was recognized that the photon must be accompanied by a second massive neutral vector boson, the $\mathrm{Z}^{0}$ particle. We now wish to appeal to this historical precedent. Let us imagine that 
the pion, like the photon, is actually a linear combination of two "ur-fields." For the photon these ur-fields are the hypercharge gauge field $Y_{\underline{a}}$ and the third component of isospin gauge field $W_{\underline{a}}^{3}$.

To play the role of the ur-fields for the pion we introduce two pseudo-scalar fields denoted by $A^{i}(x)$ and $B^{i}(x)$

$$
\begin{aligned}
& A^{i}(x)=\left[\Pi^{i}(x) \cos \left(\gamma_{\mathrm{S}}\right)+\Theta^{i}(x) \sin \left(\gamma_{\mathrm{S}}\right)\right], \\
& B^{i}(x)=\left[-\Pi^{i}(x) \sin \left(\gamma_{\mathrm{S}}\right)+\Theta^{i}(x) \cos \left(\gamma_{\mathrm{S}}\right)\right] .
\end{aligned}
$$

We imagine that the pseudo-scalar spin-0 fields $\Pi^{i}$ and $\Theta^{i}$ are the "physical states" with the latter playing the role of the $\mathrm{Z}^{0}$-particle. It is obvious that the quantity $\gamma_{S}$ here plays the analogous role of the weak mixing angle, $\theta_{W}$.

The next problem to confront is that of to what representation of the $S U_{L}(3) \otimes$ $S U_{R}(3)$ symmetry should $A^{i}$ and $B^{i}$ belong? Following the precedent of the GlashowWeinberg-Salam model wherein $Y_{\underline{a}}$ and $W_{\underline{a}}^{3}$ are assigned to distinct representations of $S U_{W}(2) \otimes U_{Y}(1)$, we assign the pseudoscalar ur-fields to distinct representations. We do this by assuming that $A^{i}$ transforms like a coordinate of the Lie algebra manifold but that $B^{i}$ transforms like a 1-form. This statement implies that the transformation laws for $A^{i}$ are obtained by simply replacing $\Pi^{i} \rightarrow A^{i}$ in (2-4) above. It is convenient to also make a slight re-definition in how the group elements $U$ are obtained from the Lie algebra element $A^{i}$,

$$
U(A) \equiv \exp \left[\frac{i A^{i} t_{i}}{f_{\pi} \cos \left(\gamma_{\mathrm{S}}\right)}\right],
$$

For $B^{i}$ however, the finite transformation law must take the form

$$
\left(B^{i}\right)^{\prime}=\left(\partial K^{i}(A) / \partial A^{j}\right) B^{j}
$$

or infinitesimally as

$$
\delta B^{i}=\alpha^{(A)}\left(\partial \xi_{(A)}^{i}(A) / \partial A^{j}\right) B^{j}
$$

Finally given these assignments of transformation properties, an action that is invariant takes the form,

$$
\mathcal{S}_{\sigma}(A, B)=-\left(\frac{f_{\pi}^{2}}{2 C_{2}}\right) \cos ^{2}\left(\gamma_{S}\right) \int d^{4} x \operatorname{Tr}\left[\mathcal{N}_{0}\left(\partial^{\underline{a}} U^{-1}\right)\left(\partial_{\underline{a}} U\right)+\mathcal{N}_{1}\left(\partial^{\underline{a}} \widetilde{B}^{\dagger}\right)\left(\partial_{\underline{a}} \widetilde{B}\right)\right]
$$

in terms of the matrix field $\widetilde{B} \equiv i\left(\partial U / \partial A^{i}\right) B^{i}$ and normalization constants $\mathcal{N}_{0}, \mathcal{N}_{1}$. In order to fix these normalization constants, we note that

$$
\partial_{\underline{a}} U=\left(\frac{\partial U}{\partial A^{i}}\right)\left(\partial_{\underline{a}} A^{i}\right) \equiv\left(\partial_{i} U\right)\left(\partial_{\underline{a}} A^{i}\right) \quad, \quad \partial^{\underline{a}} U^{-1}=\left(\partial_{i} U^{-1}\right)\left(\partial^{\underline{a}} A^{i}\right),
$$




$$
\rightarrow \operatorname{Tr}\left[\left(\partial^{\underline{a}} U^{-1}\right)\left(\partial_{\underline{a}} U\right)\right]=\operatorname{Tr}\left[\left(\partial_{i} U^{-1}\right)\left(\partial_{j} U\right)\right]\left(\partial^{\underline{a}} A^{i}\right)\left(\partial_{\underline{a}} A^{j}\right)
$$

In a similar manner, we find,

$$
\begin{aligned}
\operatorname{Tr}\left[\left(\partial^{\underline{a}} \widetilde{B}^{\dagger}\right)\left(\partial_{\underline{a}} \widetilde{B}\right)\right]= & \operatorname{Tr}\left[\left(\partial_{i} U^{-1}\right)\left(\partial_{j} U\right)\right]\left(\partial^{\underline{a}} B^{i}\right)\left(\partial_{\underline{a}} B^{j}\right) \\
& +\operatorname{Tr}\left[\left(\partial_{i} U^{-1}\right)\left(\partial_{j} \partial_{k} U\right)\right]\left(\partial^{\underline{a}} B^{i}\right)\left(\partial_{\underline{a}} A^{j}\right) B^{k} \\
& +\operatorname{Tr}\left[\left(\partial_{j} \partial_{k} U^{-1}\right)\left(\partial_{i} U\right)\right]\left(\partial^{\underline{a}} B^{i}\right)\left(\partial_{\underline{a}} A^{j}\right) B^{k} \\
& +\operatorname{Tr}\left[\left(\partial_{i} \partial_{j} U^{-1}\right)\left(\partial_{k} \partial_{l} U\right)\right]\left(\partial^{\underline{a}} A^{i}\right) B^{j}\left(\partial_{\underline{a}} A^{k}\right) B^{l}
\end{aligned}
$$

where we have used the identity $U^{\dagger}=U^{-1}$. It is also useful to note that in (11) and all subsequent equations

$$
\partial_{i}=\frac{\partial}{\partial A^{i}}=\cos \left(\gamma_{S}\right) \frac{\partial}{\partial \Pi^{i}}+\sin \left(\gamma_{S}\right) \frac{\partial}{\partial \Theta^{i}}
$$

Now the "physics" of the pions described by the action in (5) is different from that described in (10). It is of course of interest to know explicitly how such differences might manifest themselves for example in amplitudes. In order to see this, we will first consider (10) in the limit where $\Theta=0$ and calculate the resulting action. This is summarized by the following equation,

$$
\widetilde{\mathcal{S}}_{\sigma}(\Pi) \equiv\left[\lim _{\Theta \rightarrow 0} \mathcal{S}_{\sigma}(A, B)\right]=\mathcal{S}_{\sigma}(\Pi)+\sin ^{2}\left(\gamma_{S}\right)\left[S_{1}(\Pi)+S_{2}(\Pi)\right],
$$

where we have chosen to set $\mathcal{N}_{0}=\mathcal{N}_{1}=1$. The explicit forms of the actions $S_{1}$ and $S_{2}$ are given by

$$
\begin{aligned}
& S_{1}(\Pi)=-\left(\frac{f_{\pi}^{2}}{2 C_{2}}\right) \int d^{4} x \operatorname{Tr}\left[\left(\frac{\partial U^{\dagger}}{\partial \Pi^{2}}\right)\left(\frac{\partial^{2} U}{\partial \Pi^{\jmath} \partial \Pi^{k}}\right)+\text { h.c. }\right] \Pi^{i}\left(\partial^{\underline{a}} \Pi^{j}\right)\left(\partial_{\underline{a}} \Pi^{k}\right), \\
& S_{2}(\Pi)=-\left(\frac{f_{\pi}^{2}}{2 C_{2}}\right) \int d^{4} x \operatorname{Tr}\left[\left(\frac{\partial^{2} U^{\dagger}}{\partial \Pi^{2} \partial \Pi^{j}}\right)\left(\frac{\partial^{2} U}{\partial \Pi^{k} \partial \Pi^{t}}\right)\right] \Pi^{i} \Pi^{k}\left(\partial^{\underline{a}} \Pi^{j}\right)\left(\partial_{\underline{a}} \Pi^{l}\right) .
\end{aligned}
$$

In arriving at these results we have used

$$
\begin{aligned}
& \lim _{\Theta \rightarrow 0} U(A)=U(\Pi) \quad, \quad \lim _{\Theta \rightarrow 0}\left(\frac{\partial}{\partial A^{i}}\right)=\sec \left(\gamma_{S}\right)\left(\frac{\partial}{\partial \Pi^{i}}\right), \\
& \lim _{\Theta \rightarrow 0} A^{i}=\Pi^{i} \cos \left(\gamma_{S}\right), \lim _{\Theta \rightarrow 0} B^{i}=-\Pi^{i} \sin \left(\gamma_{S}\right) .
\end{aligned}
$$

A few comments about the second limit above are in order. Since the operations of taking the limit as $\Theta \rightarrow 0$ and $\frac{\partial}{\partial \Theta^{i}}$ do not commute, we must take some care. Due to the functional form of $U(A)$, the following operator equation is valid when acting on it or any of its derivatives w.r.t. $A^{i}$,

$$
\frac{\partial}{\partial \Theta^{i}}=\tan \left(\gamma_{S}\right) \frac{\partial}{\partial \Pi^{i}}
$$


When this is inserted into (14) we arrive at the second limit stated in (17).

It is easily seen that the differences in contributions to pure pion amplitudes calculated using the two distinct actions ((5) vs. (10)) will be at least of order $\sin ^{2}\left(\gamma_{S}\right)$. For $\gamma_{S} \sim \frac{1}{10}$ this corresponds to a suppression by a factor of one hundred between the differences of amplitudes computed from the two different actions. In principle at least (although we are pessimistic about the practicality of this), precision pion physics experiments should place upper limits on this parameter. Additional suppression is implied by the reciprocal powers of $f_{\pi}$ that are implicitly contained in calculating $\left(\partial U / \partial A^{i}\right)$ and $\left(\partial^{2} U / \partial A^{i} \partial A^{j}\right)$.

The process of taking the limit at $\Theta \rightarrow 0$ has one other important consequence. Although the two terms in (10) are separately $S U_{L}(3) \otimes S U_{R}(3)$ invariant, after taking the limit, the resultant action of (15) breaks this symmetry by terms of order $\sin ^{2}\left(\gamma_{S}\right)$. One way to see that the symmetry is broken is to note that the variation of $\Theta^{i}$ is given by

$$
\delta \Theta^{i}=-i f_{\pi} \cos \left(\gamma_{S}\right) \alpha^{(A)}\left\{\sin \left(\gamma_{S}\right) \xi_{(A)}^{i}+\cos \left(\gamma_{S}\right) B^{k}\left(\partial_{k} \xi_{(A)}^{i}\right)\right\},
$$

and if we take the limit of this equations as $\Theta \rightarrow 0$ then there arises the constraint $\gamma_{S}=\frac{\pi}{2} n$ where $n$ is any integer. In other words, no mixing in the initial model.

It is easily possible to work out, to any order, the coupling of the hypothetical " $\Theta$-pion" $S U(3)$ multiplet to the usual pion $S U(3)$ multiplet. Due to the rigid restrictions imposed by the $S U_{L}(3) \otimes S U_{R}(3)$ geometry, these couplings are completely determined. However, once more in analogy to the electroweak paradigm, we would expect there to be a large mass gap between these (even if they existed as bound states of sufficient lifetimes to be detectable) and the usual pion octet that would defeat the naive hope of detection. To lowest order in the $\Theta$-pion field, the couplings that follow from (10) take the form

$$
\begin{aligned}
& \mathcal{S}_{1^{\text {st } \text { order int. }}}(\Theta)=-\left(\frac{f_{\pi}^{2}}{2 C_{2}}\right) \int d^{4} x\left\{\Theta^{i}\left[\mathcal{T}_{i}-\left(\partial^{\underline{a}} \mathcal{V}_{\underline{a} i}\right)\right]\right\} \\
& \mathcal{T}_{p}=g_{1} \operatorname{Tr}\left[\frac{\partial}{\partial \Pi^{p}}\left[\left(\frac{\partial U^{\dagger}}{\partial \Pi^{i}}\right)\left(\frac{\partial U}{\partial \Pi^{j}}\right)\right]\right]\left(\partial^{\underline{a} \Pi^{i}}\right)\left(\partial_{\underline{a}} \Pi^{j}\right) \\
& +g_{1} \operatorname{Tr}\left[\frac{\partial}{\partial \Pi^{p}}\left[\left(\frac{\partial U^{\dagger}}{\partial \Pi^{i}}\right)\left(\frac{\partial^{2} U}{\partial \Pi^{\jmath} \partial \Pi^{k}}\right)+\text { h. c. }\right]\right]\left(\partial^{\underline{a}} \Pi^{i}\right)\left(\partial_{\underline{a}} \Pi^{j}\right) \Pi^{k} \\
& -g_{2} \operatorname{Tr}\left[\left[\left(\frac{\partial^{2} U^{\dagger}}{\partial \Pi^{2} \partial \Pi^{j}}\right)\left(\frac{\partial^{2} U}{\partial \Pi^{k} \partial \Pi^{p}}\right)+\text { h. c. }\right]\right]\left(\partial^{\underline{a}} \Pi^{i}\right) \Pi^{j}\left(\partial_{\underline{a}} \Pi^{k}\right) \\
& +g_{1} \operatorname{Tr}\left[\frac{\partial}{\partial \Pi^{p}}\left[\left(\frac{\partial^{2} U^{\dagger}}{\partial \Pi^{2} \partial \Pi^{j}}\right)\left(\frac{\partial^{2} U}{\partial \Pi^{k} \partial \Pi^{t}}\right)\right]\right]\left(\partial^{a} \Pi^{i}\right) \Pi^{j}\left(\partial_{\underline{a}} \Pi^{k}\right) \Pi^{l},
\end{aligned}
$$




$$
\begin{aligned}
\mathcal{V}_{\underline{a} i}= & g_{2} \operatorname{Tr}\left[\left(\frac{\partial U^{\dagger}}{\partial \Pi^{2}}\right)\left(\frac{\partial^{2} U}{\partial \Pi^{\jmath} \partial \Pi^{k}}\right)+\text { h. c. }\right]\left(\partial_{\underline{a}} \Pi^{j}\right) \Pi^{k} \\
& -g_{1} \operatorname{Tr}\left[\left(\frac{\partial U^{\dagger}}{\partial \Pi^{j}}\right)\left(\frac{\partial^{2} U}{\partial \Pi^{2} \partial \Pi^{k}}\right)+\text { h.c. }\right]\left(\partial_{\underline{a}} \Pi^{j}\right) \Pi^{k} \\
& +g_{1} \operatorname{Tr}\left[\left(\frac{\partial^{2} U^{\dagger}}{\partial \Pi^{2} \partial \Pi^{j}}\right)\left(\frac{\partial^{2} U}{\partial \Pi^{k} \partial \Pi^{t}}\right)+\text { h. c. }\right] \Pi^{j}\left(\partial_{\underline{a}} \Pi^{k}\right) \Pi^{l},
\end{aligned}
$$

where the constants $g_{i}$ are defined by

$$
g_{1}=\sin ^{2}\left(\gamma_{S}\right) \tan \left(\gamma_{S}\right), g_{2}=\sin \left(\gamma_{S}\right) \cos \left(\gamma_{S}\right)
$$

The derivation of (20) follows from a Taylor expansion w.r.t $\Theta^{i}$ and the use of (18). We note that $g_{1}=O\left[\left(\gamma_{S}\right)^{3}\right]$ and $g_{2}=O\left[\left(\gamma_{S}\right)\right]$ so that the leading vertex for the emission of the $\Theta$-pion is obtained from the first term of $\mathcal{V}_{\underline{a} i}$. The next such vertex is obtained from the third term in $\mathcal{T}_{i}$. We emphasize that the group elements $U$ that appear in (20) are evaluated in the limit of $\Theta \rightarrow 0$.

Having understood this simple mechanism for describing the pion in a manner where it is a linear combination of two distinct representations of the $S U_{L}(3) \otimes S U_{R}(3)$ group manifold, it should be natural to ask, if it possible to have even more complicated descriptions of the pion. The answer is yes. The key point is that higher order tensor fields $B^{i_{1} \ldots i_{p}}$ over the $S U_{L}(3) \otimes S U_{R}(3)$ group manifold possess infinitesimal variations of the form

$$
\delta B^{i_{1} \ldots i_{p}}=\alpha_{1}^{(A)} \cdots \alpha_{p}^{(A)}\left(\partial \xi_{(A)}^{i_{1}} / \partial A^{j_{1}}\right) \cdots\left(\partial \xi_{(A)}^{i_{p}} / \partial A^{j_{p}}\right) B^{j_{1} \ldots j_{p}}
$$

Multiplying by factors of $\left(\partial U / \partial A^{i}\right)$ and $\left(\partial U^{\dagger} / \partial A^{i}\right)$ appropriately, such higher tensor fields can be converted into matrix valued fields whose actions take precisely the same form as the last term in (10). Clearly linear combinations of such actions lead to even more complicated descriptions and many more mixing angles.

The observations that we have made in this section amount to mathematical curiosities. There is no particular motivation for choosing any of the ur-formulations of the pion over the standard one. The presence of supersymmetric models with Dirac-like pionini has the potential to change this dramatically.

\section{Chiral Superfield Fibers Over Chiral Superfield Manifolds}

In the third work of ref. [1], the general $\sigma$-model geometry of a CNM model was introduced. The distinctive feature that differentiates this from the usual $4 \mathrm{D}, \mathrm{N}=$ 
1 supersymmetric non-linear $\sigma$-model geometry is that the nonminimal superfields can be introduced as 1-forms not coordinates of the $\sigma$-model manifold. However, once this geometrical feature is realized, it becomes obvious that in a general chiral superfield non-linear $\sigma$-model with coordinates $\left(\Phi^{1}, \Phi^{2}, \ldots, \Phi^{2 p}\right)$, it is always possible to regard a subset (say $\left(\Phi^{1}, \Phi^{2}, \ldots, \Phi^{p}\right)$ ) as the coordinates of a sub-manifold and replace the remaining chiral superfields by 1 -forms over the sub-manifold. For an $S U_{L}(3) \otimes S U_{R}(3)$ group manifold (which is what we require in the present setting) this idea may be implemented in the following way.

It is a well known fact that in order to describe a Dirac spinor requires two distinct superfields. So if the pionini existed as Dirac particles, two $S U(3)$ octets of chiral superfields are required. We may denote these by $\Phi_{R}^{\mathrm{I}}$ and $\Phi_{L}^{\mathrm{I}}$ with $\mathrm{I}=1, \ldots, 8$. Let us for the moment assume that both transform as the coordinate of the $S U_{L}(3) \otimes S U_{R}(3)$ group manifold. If this is the case, we may exponentiate appropriately either one to form an $S U(3)$ group element. For this purpose let us pick $\Phi_{R}^{\mathrm{I}}$ so that $U\left(\Phi_{R}\right)$ is such a group element. We may define a new chiral superfield variable $\chi^{\mathrm{K}}$ though the equation $\Phi_{L}^{\mathrm{I}} \equiv C_{2}{ }^{-1} \operatorname{Tr}\left[\left(\partial_{\mathrm{K}} U\left(\Phi_{R}\right)\right) \chi^{\mathrm{K}} t^{\mathrm{I}}\right]$. As a consequence of this definition, $\chi^{\mathrm{I}}$ must be a 1-form over the group manifold. Thus, an equivalent set of variables is given by $\Phi_{R}^{\mathrm{I}}$ and $\chi^{\mathrm{I}}$. In this way Dirac-like pionini leads to the introduction of chiral superfields that are distinct representations of the group manifold.

Once one is forced to utilize two chiral superfields, then the pion might occur as a linear combination of the two psuedo-scalar component fields that occur at leading order in the $\theta$-expansion. This leads to the introduction of a mixing angle and thus the simplest ur-formulation, as presented in section two, seems to arise whenever we demand the presence of Dirac-like pionini. This is independent of whether we utilize two chiral superfields or one chiral superfield and one nonminimal superfield as in the CNM models.

So we may begin by introducing a set of chiral superfields $\Phi^{\mathrm{I}}$ that correspond to the coordinates of the $S U(3)$ Lie-algebra. Next we introduce a distinct set of $S U(3)$ 1-form chiral superfields $\chi^{\mathrm{I}}$. The infinitesimal transformation law of $\Phi^{\mathrm{I}}$ is now determined by (3) replacing all ordinary fields by superfields. Similarly, the infinitesimal transformation laws of $\chi^{\mathrm{I}}$ are determined by (8) where again all ordinary fields are replaced by superfields.

Upon defining the superfield group elements $U$ by

$$
U(\Phi) \equiv \exp \left[\frac{\Phi^{\mathrm{I}} t_{\mathrm{I}}}{f_{\pi} \cos \left(\gamma_{\mathrm{S}}\right)}\right],
$$


we may take for an action国,

$$
\begin{aligned}
\mathcal{S}(\Phi, \chi)=\left(\frac{f_{\pi}^{2}}{C_{2}}\right) \cos ^{2}\left(\gamma_{S}\right) & \int d^{4} x d^{2} \theta d^{2} \bar{\theta}\left\{\left(\mathcal{N}_{0} \operatorname{Tr}\left[U^{\dagger} U\right]+\mathcal{N}_{1} \operatorname{Tr}\left[\hat{\chi}^{\dagger} \hat{\chi}\right]\right)\right. \\
- & {\left[i \mathcal{H}_{\mathrm{I} \overline{\mathrm{J} K L}}^{1}(\Phi, \bar{\Phi}) \chi^{\mathrm{I}}\left(\bar{D}^{\dot{\beta}} \bar{\Phi}^{\mathrm{J}}\right)\left(D^{\gamma} \Phi^{\mathrm{K}}\right)\left(\partial_{\gamma \dot{\beta}} \Phi^{\mathrm{L}}\right)+\text { h.c. }\right] } \\
- & {\left.\left[i \mathcal{H}_{\overline{\mathrm{I}} \overline{\mathrm{J} K \mathrm{~L}}}^{2}(\Phi, \bar{\Phi}) \bar{\chi}^{\mathrm{I}}\left(\bar{D}^{\dot{\beta}} \bar{\Phi}^{\mathrm{J}}\right)\left(D^{\gamma} \Phi^{\mathrm{K}}\right)\left(\partial_{\gamma \dot{\beta}} \Phi^{\mathrm{L}}\right)+\text { h.c. }\right]\right\} } \\
& +\mathcal{S}_{N R}(\Phi)
\end{aligned}
$$

where $S_{N R}$ refer to the Rohm-Nemanschansky action [10. Due to the work of these authors, we know that the ultimate term above contains a component level WZNW term. With a little modification of their calculations, it is easy to see that third and fourth actions above also contain terms that may be interpreted as WZNW terms. So for arbitrary values of $\gamma_{S}$, this action always contains a component level WZNW term. Finally we note that the action in (24) does not correspond to the dual formulation of the CNM models of [2]. This is most easily verified by noting that only one factor of $\chi$ appears in the WZNW terms above. The dual CNM models always are quadratic in this dependence. By the same reasoning, we see that the NR action is also not the dual CNM model action.

The $S U_{L}(3) \otimes S U_{R}(3)$ symmetry of this action is guaranteed as long as $\mathcal{H}_{\mathrm{I} \overline{\mathrm{J}} \mathrm{L}}^{1}$ and $\mathcal{H}_{\overline{\mathrm{I}} \overline{\mathrm{J} K L}}^{2}$ are appropriately transforming tensors, i.e. respectively of type $(3,1)$ and $(2,2)$ with respect to a holomorphic-anti-holomorphic form basis.

On the other hand, the first two terms in (24) obviously describe a particular 4D, $\mathrm{N}=1$ supersymmetric non-linear $\sigma$-model with a Kähler geometry. We now want to focus on the component field action described by these terms. First we define the component field via the following definitions (with $\Phi \equiv \Phi^{\mathrm{I}} t_{\mathrm{I}}$ ),

$$
\begin{gathered}
\Phi \mid \equiv A^{\mathrm{I}}(x) t_{\mathrm{I}}=\left\{\mathcal{A}^{\mathrm{I}}(x)+i\left[\Pi^{\mathrm{I}}(x) \cos \left(\gamma_{\mathrm{S}}\right)+\Theta^{\mathrm{I}}(x) \sin \left(\gamma_{\mathrm{S}}\right)\right]\right\} t_{\mathrm{I}}, \\
\left(D_{\alpha} U\right)\left|\equiv \psi_{\alpha}(x) \quad, \quad\left(D^{2} U\right)\right| \equiv \mathrm{F}(x),
\end{gathered}
$$

where we observe that upon setting $\mathcal{A}^{\mathrm{I}}=0$, this reduces back to (7). In a similar manner we define,

$$
\begin{aligned}
\widehat{\chi} \mid \equiv \widehat{B}(x) & =\left[\partial_{\mathrm{I}} U(\Phi \mid)\right]\left\{\mathcal{B}^{\mathrm{I}}(x)+i\left[-\Pi^{\mathrm{I}}(x) \sin \left(\gamma_{\mathrm{S}}\right)+\Theta^{\mathrm{I}}(x) \cos \left(\gamma_{\mathrm{S}}\right)\right]\right\}, \\
\left(D_{\alpha} \widehat{\chi}\right) \mid & \equiv \zeta_{\alpha}(x), \quad\left(D^{2} \widehat{\chi}\right) \mid \equiv \mathrm{G}(x) .
\end{aligned}
$$

\footnotetext{
${ }^{5}$ For the sake of simplicity, we will ignore all other higher derivative terms as well as the the issue of mass terms.
} 
where $\partial_{\mathrm{I}} U(\Phi \mid)=\left(\partial U(\Phi \mid) / \partial A^{\mathrm{I}}\right)$. Note that the component denoted by $\widehat{B}$ here is slightly different from the similar field defined in (10). Only upon setting $\mathcal{A}^{\mathrm{I}}=\mathcal{B}^{\mathrm{I}}=0$ will the two fields coincide.

After a direct calculation we find,

$$
\begin{aligned}
& \left(\frac{f_{\pi}^{2}}{C_{2}}\right) \cos ^{2}\left(\gamma_{S}\right) \int d^{4} x d^{2} \theta d^{2} \bar{\theta}\left(\mathcal{N}_{0} \operatorname{Tr}\left[U^{\dagger} U\right]+\mathcal{N}_{1} \operatorname{Tr}\left[\widehat{\chi}^{\dagger} \widehat{\chi}\right]\right)= \\
& \quad\left(\frac{f_{\pi}^{2}}{C_{2}}\right) \mathcal{N}_{0} \cos ^{2}\left(\gamma_{S}\right) \int d^{4} x \operatorname{Tr}\left[-\frac{1}{2}\left[\partial^{\underline{a}} U^{\dagger}(\Phi \mid)\right]\left[\partial_{\underline{a}} U(\Phi \mid)\right]-i \bar{\psi}^{\dot{\alpha} \dagger} \partial_{\underline{a}} \psi^{\alpha}+\mathrm{F}^{\dagger} \mathrm{F}\right] \\
& +\left(\frac{f_{\pi}^{2}}{C_{2}}\right) \mathcal{N}_{1} \cos ^{2}\left(\gamma_{S}\right) \int d^{4} x \operatorname{Tr}\left[-\frac{1}{2}\left(\partial^{\underline{a}} \widehat{B}^{\dagger}\right)\left(\partial_{\underline{a}} \widehat{B}\right)-i \zeta^{\alpha} \partial_{\underline{a}} \bar{\zeta}^{\dot{\alpha} \dagger}+\mathrm{G}^{\dagger} \mathrm{G}\right] .
\end{aligned}
$$

Using the same methods as were developed in the discussion of the $\Theta$-pion multiplet coupling to the usual pion multiplet in section two, similar results can be derived from (27) for the coupling to the $\mathcal{A}$ and $\mathcal{B}$ multiplets. As well it is straightforward to see that using the definitions above, the Dirac pionino defined by

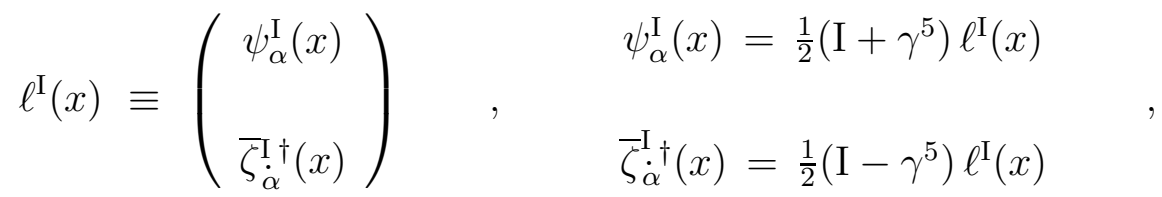

is a free field (at this level) with interactions only arising through the WZNW terms in (24). Thus, the fermionic terms of (27) can be combined as

$$
i \bar{\ell} \not \partial \ell=i \bar{\psi}^{\dot{\alpha} \dagger} \partial_{\underline{a}} \psi^{\alpha}+i \zeta^{\alpha} \partial_{\underline{a}} \bar{\zeta}^{\dot{\alpha} \dagger}
$$

Although the sumb in (29) is invariant under the exchange of $\psi_{\alpha}$ and $\bar{\zeta}_{\dot{\alpha}}^{\dagger}$, (thus insuring parity invariance) the total action in (24) does not possess this symmetry. Thus, we find the result that the mere presence of Dirac-like pionini implies a breaking of parity even in the absence of the electroweak interaction. The breaking of parity can also be observed in the form of the supersymmetry variations that leave the action invariant. The left and right components of $\ell$ enter these variations in a highly asymmetrical manner. A similar result has been observed within the CNM models. Although the kinetic energy terms for the spin-0 fields in (27) appear to be the same as that in (10), this is only an apparent similarity. The terms in (27) include kinetic energies for $\mathcal{A}^{\mathrm{I}}$ and $\mathcal{B}^{\mathrm{I}}$ which do not appear at all in (10). Similarly, the field $\mathcal{A}^{\mathrm{I}}$ appears to all powers in (27) while $\mathcal{B}^{\mathrm{I}}$ appears only quadratically. This nonpolynomial appearance of $\mathcal{A}^{\mathrm{I}}$ can be understood upon observing that $U^{\dagger}(\Phi \mid) \neq U(\Phi \mid)$ which implies that the

\footnotetext{
${ }^{6}$ By further field re-definitions, the spinor action in (27) can be made to possess a canonical normalization.
} 
sigma model in (27) possesses a non-compact geometry in contrast to the compact geometry described by the model in (10). One of the most obvious points is that if we only retain the fields $\Pi^{i}$ and $\Theta^{i}$ in (27) and set all remaining fields to zero, then the superfield action in (27) reduces to the action in (10), the pure pion limit of which is

given by $\widetilde{\mathcal{S}}_{\sigma}(\Pi)$ defined in (15). By the same duality argument used in [2], this must also be the pure pion limit of the CNM $\sigma$-model of the supersymmetric QCD effective action (after eliminating all auxiliary fields).

\section{Conclusion}

As we saw in the beginning of this work, the ur-formulations of the $S U_{L}(3) \otimes$ $S U_{R}(3)$ geometry of the pion exist outside of the supersymmetric context. However, without supersymmetry these are very ad hoc and unnatural. In the presence of Dirac pionini this is not the case. As mentioned already the simplest ur-formulation also occurs in the context of our previously constructed CNM models [1, 2]. The model in (10) is determined (up to constants) totally by the $S U_{L}(3) \otimes S U_{R}(3)$ geometry not supersymmetry.

This observation permits us to quantify the statement made in ref. [2], that "..., the manifestly supersymmetric action should come as close as possible to being in agreement with the Gasser-Leutwyler parametrization [11]." The mixing angle $\gamma_{S}$ is a measure of this closeness. The smaller the value of this angle, the closer the ur-formulation $\left(\widetilde{\mathcal{S}}_{\sigma}\right)$ contained in the CNM model comes to exact agreement with the usual model of pure pion physics $\left(\mathcal{S}_{\sigma}\right)$. More remarkably, the CNM model is also the only one that requires the mixing angle $\gamma_{S}$ to be non-zero on theoretical grounds. Thus, we find the remarkable situation that the parameter $\gamma_{S}$ (assuming it is positive) must be bounded (by an equation like $0<\gamma_{S}<\frac{1}{10}$ ) from above by experimental viability of the model and from below by the fact that theoretically it must contain a pion.

As we have seen in a hypothetical world of unbroken supersymmetry, it is also possible to describe Dirac pionini with the sole use of chiral superfields. However, in the purely chiral superfield formulation of such a theory, all possible values of the mixing angle $\gamma_{S}$ are allowed theoretically. Thus, we may say that the mixing angle $\gamma_{S}$ is essential within the context of the CNM model but is not otherwise. The concept of essential versus inessential mixing angles can also be seen within the standard model. The weak mixing angle $\left(\theta_{W}\right)$ is an essential mixing angle because there are certain values of $\theta_{W}$ that are not allowed on purely theoretical grounds (i.e. $\sin \left(2 \theta_{W}\right) \neq 0$ ). 
On the other hand, the Cabibbo and Kobayashi-Maskawa angles are examples of inessential mixing angles.

So it looks like there are many distinct ways to construct $4 \mathrm{D}, \mathrm{N}=1$ supersymmetric extensions of the chiral model that contains the pion and Dirac pionini. This is true at least if we only require superactions that contain a $\sigma$-model term as well as a WZNW term. $\mathrm{G}$ We note, however, that the CNM model and its dual are the only ones where the transcedental functions that determine the entire pion sector of the supersymmetric QCD effective action via the Gasser-Leutwyler parametrization, are holomorphic functions.

Finally, it is interesting that in our hypothetical universe of ur-formulations, the

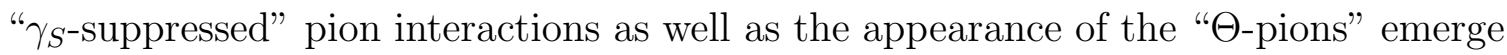
as signals in the simplest such model. These features are necessarily consequences of the $4 \mathrm{D}, \mathrm{N}=1$ supersymmetric CNM model of the the QCD effective action as well. Since supersymmetry (if it applies at all to our world) is badly broken, it is unlikely that we could detect the purely pion physics 'signals' of supersymmetry in Nature...except by the most remarkable serendipity.

\section{Acknowledgment;}

SJG wishes to thank M. Roček for conversations that led to consideration of this problem. Additional helpful comments by Thomas Cohen are gratefully recognized.

\section{References}

[1] S. J. Gates, Jr., Phys. Lett. 365B (1996) 132; idem. "A New Proposed Description of the $4 \mathrm{D}, \mathrm{N}=1$ Supersymmetric Effective Action for Scalar Multiplets" to appear in the proceedings of the Second International Sakharov Conference on Physics, May 20-25, Moscow, Russia; idem. Univ. of Maryland Preprint, UMDEPP 96-99, to appear in Nucl. Phys. B.

[2] S. J. Gates, Jr., M. T. Grisaru, M. E. Knutt-Wehlau, M. Roček and O. A. Soloviev, "N=1 Supersymmetric Extension of the QCD Effective Ac-

\footnotetext{
${ }^{7}$ It should be noted that it is only in the case of the CNM models that it has been verified that all of the terms of the Gasser-Leutwyler parametrization can be extended into fully supersym -metric analogs. So for example, it is not known whether there even exist an auxiliary-free description of the ' $L_{1}$ term' using only chiral superfields.
} 
tion," Univ. of Maryland Preprint UMDEPP 97-27, hep-th/9612196, to appear in Phys. Lett. B.

[3] J. Donoghue, E. Golowich and B. Holstein, Dynamics of the Standard Model, Cambridge Monographs on Particle Physics, Nuclear Physics and Cosmology, (Cambridge Univ. Press, 1992), J. D. Walecka, Theoretical Nuclear and Subnuclear Physics, (Cambridge Univ. Press, 1992); S. Weinberg, The Quantum Theory of Fields, Vol. II, (Cambridge Univ. Press, 1996).

[4] D. Amati, K. Konishi, Y. Meurice, G.C. Rossi and G. Veneziano, "NonPerturbative Aspects in Supersymmetric Gauge Theories," Physics Reports 162, 4 (1988) 169-248.

[5] S. V. Ketov, "Solitons, Monopoles and Duality: from Sine-Gordon to SeibergWitten," DESY 96-244, ITP-UH-23/96 (ITP, University of Hannover), hepth/9611209.

[6] J. Wess and B. Zumino, Nucl. Phys. B70 (1974) 39.

[7] S. J. Gates, Jr. and W. Siegel, Nucl. Phys. B187 (1981) 389; S. J. Gates, Jr., M.T. Grisaru, M. Roček and W. Siegel, Superspace Benjamin Cummings, (1983) Reading, MA., pp. 148-157, 199-200; B. B. Deo and S. J. Gates, Jr., Nucl. Phys. B254 (1985) 187.

[8] See for example R. N. Mohapatra, Unification and Supersymmetry, SpringerVerlag, New York (1986); G. Kane, Modern Elementary Particle Physics, Addison-Wesley Pub. Co., Reading, MA (1993) and references therein.

[9] S. L. Glashow, Nucl. Phys.22 (1961) 579, A. Salam and J.C. Ward, Phys. Lett. 13 (1964) 168; S. Weinberg, Phys. Rev. Lett. 19 (1967) 1264; A. Salam, in Elementary Particle Theory (edited by N. Svartholm) Almquist and Forlag, Stockholm, 1968.

[10] J. Wess and B. Zumino, Phys. Lett. 37B (1971) 95; S. Novikov, Sov. Math. Dokl. 24 (1981) 222; E. Witten, Nucl. Phys. B223 (1983) 422; T. E. Clark and S. T. Love, Phys. Lett. 138B (1984) 289; D. Nemeschansky and R. Rohm, Nucl. Phys. B249 (1985) 157.

[11] J. Gasser and H. Leutwyler, Nucl. Phys. B250 (1985) 465. 